\title{
Consideraciones generales respecto a la necesidad de practicar una pedagogía sobre la finitud humana en la educación formal. Estudio de caso
}

\author{
Anabel Ramos Pla \\ Ramon Camats i Guàrdia \\ Universitat de Lleida. España. \\ anabelrms@gmail.com \\ rcamats@pip.udl.cat
}

Recibido: 26/9/2016

Aceptado: 13/11/2016

Publicado: 26/10/2017

\section{Resumen}

Actualmente, la pedagogía de la muerte es un tema formativo emergente, sobre todo en el ámbito español. Asimismo, la finitud humana continúa siendo un tabú que cuesta derribar, tanto socialmente como pedagógicamente. En contraposición a dicha circunstancia, apostamos por la necesidad de tratar la pedagogía de la finitud humana desde la conciencia de la muerte en el ámbito de la didáctica formal. La aportación presenta un estudio de caso realizado en dos centros docentes de Lleida (España), donde los profesionales de la educación, la psicología, la pedagogía y/o la psicopedagogía respondieron un cuestionario diseñado ex profeso. Los resultados del cuestionario y de las entrevistas llevadas a cabo reflejan que los enseñantes no se sienten suficientemente formados ni preparados para realizar acciones pedagógicas respecto a situaciones infantiles y juveniles de muerte y de duelo. Se apuesta, finalmente, por la formación docente específica y se proponen una serie de estrategias generales para poder afrontar dicha temática en contextos de educación formal. De este modo, se pretende romper el tabú al cual se enfrenta la muerte en la profesión docente y psicopedagógica.

Palabras clave: educación para la muerte; concepto de muerte; inteligencia emocional; duelo; soporte a la educación primaria

Resum. Consideracions generals respecte a la necessitat de practicar una pedagogia sobre la finitud humana en l'educació formal. Estudi de cas

Actualment, la pedagogia de la mort és un tema formatiu emergent, sobretot en l'àmbit espanyol. Així mateix, la finitud humana continua sent un tabú que costa derrocar, tant socialment com pedagògicament. En contraposició a aquesta circumstància, apostem per la necessitat de practicar la pedagogia de la finitud humana des de la consciència de la mort en l'àmbit de la didàctica formal. L'aportació presenta un estudi de cas realitzat en dos centres docents de Lleida, on els professionals de l'educació, la psicologia, la pedagogia i/o la psicopedagogia van respondre un qüestionari dissenyat ex professo. Els resultats del qüestionari i de les entrevistes dutes a terme reflecteixen que els ensenyants no se senten preparats per realitzar accions pedagògiques respecte a situacions infantils i juvenils de 
mort i de dol. S'aposta, finalment, por la formació docent específica i proposem una sèrie d'estratègies generals per poder afrontar aquesta temàtica en contextos d'educació formal. D'aquesta manera, es pretén trencar el tabú al qual s'enfronta la mort en la professió docent i psicopedagògica.

Paraules clau: educació per a la mort; concepte de mort; intel.ligència emocional; dol; suport a l'educació primària

Abstract. General considerations about the need for pedagogy on death in formal education. $A$ case study

The pedagogy of death is an emerging subject in education, especially in Spain. Human finitude is a taboo topic that is difficult to eradicate both socially and pedagogically. In contrast to this view, we focus on the need for training in human finitude based on the consciousness of death in the field of formal education. A case study of two schools in Lleida, Spain, was carried out. Professionals of education, psychology and/or educational psychology were administered a questionnaire designed specifically for the purpose of the study. The results of the questionnaire and interviews show that teachers feel unprepared for educational activities regarding situations of death and grieving children and youth. Finally, we advocate that teachers receive specific training and propose a series of general strategies to face death in the context of formal education in order to break the taboo of death in the teaching profession and educational psychology.

Keywords: death education; meaning of death; emotional intelligence; grief; elementary school support

\section{Sumario}

1. Justificación y fundamento

2. Pedagogía de la muerte en las escuelas

3. La necesidad de practicar una pedagogía preventiva y normalizadora sobre la finitud humana
4. Conclusiones

5. Propuestas de futuro

Referencias bibliográficas

\section{Justificación y fundamento}

El ser humano es finito por naturaleza y, aunque este hecho no le diferencie de los otros seres vivos, tenemos que tomar conciencia de que, un día u otro, moriremos. Saber esto no debería producirnos angustia o desesperación, sino serenidad para vivir intensamente el momento (Pedrero y Leiva, 2011). En este sentido, si se concibe la muerte como un hecho natural y, a su vez, como parte de la vida, su enseñanza es necesaria desde la infancia (Rodríguez Herrero, Herrán y Cortina, 2012).

Investigaciones realizadas por Otero y Soares (2012) confirman que existe un alto porcentaje de escolares que han sufrido, durante su escolarización en educación primaria, una pérdida significativa a causa de la muerte de un ser querido. En consecuencia, la profesión docente no puede ni debe eludir el 
tratamiento de la finitud humana en las aulas. En esta línea, debemos añadir que, aunque la pedagogía de la muerte sea un tema de investigación, formación e innovación emergente en el siglo XXI (Rodríguez Herrero, Herrán y Cortina, 2012; Olid Báez, 2013), todavía no existen suficientes investigaciones referentes a la temática que nos ocupa (Poch y Herrero, 2003; Herrán y Cortina, 2006; Otero y Soares, 2012). En este sentido, los profesionales de la educación deben romper con la dicotomía que separa al niño de la muerte. Evidentemente, las criaturas no perciben la muerte como los adultos, pero la sienten, la padecen y, por tanto, viven el proceso de duelo. Además, aunque las personas mayores queramos omitir la realidad de la defunción de un ser querido, los niños se percatan de que, ante un hecho de estas características, no nos comportamos «con normalidad». Es decir, saben que algo que no es bueno está pasando y que no se quiere hacerles partícipes de ello. Muestra de este hecho son las manifestaciones infantiles en sus juegos, donde recrean, así, historias y fantasías (Arnaiz, 2003; Esquerda y Agustí, 2010). Debemos recordar que mentir a los niños y a los adolescentes y que ellos acaben descubriendo la verdad puede conllevar una fuente de desconfianza hacia el adulto y, además, darles a sobreentender que las manifestaciones de dolor no están bien vistas socialmente, lo cual les puede incitar indirectamente a esconder, taponar y aguantar sus emociones.

Desde la antigüedad hasta la actualidad, la muerte y el duelo han pasado por múltiples variaciones que impactan de formas muy diversas (Ariés, 1975). La sociedad occidental de nuestros días está viviendo el tabú de la muerte de forma muy pronunciada (Arnaiz, 2003; Colomo y De Oña, 2014; Carmelo y Comas, 2014; Serra Llanas, 2014; Antich, Morey y Romero, citados en Cuadrado y Manzano, 2016; Gabarró, 2016), más que el que padeció hace unos años el tema del sexo, que se ha ido integrando a ella paulatinamente. Como sociedad occidental, estamos viviendo, así, una falta de conciencia respecto a la finitud humana, donde el único ser vivo que tiene conocimiento de su existencia, la omite. Asimismo, estamos de acuerdo con Esquerda y Agustí (2010), Rodríguez Herrero, Herrán y Cortina (2015a, 2015b) y Colomo (2016), quienes abogan que es necesario comprender que abordar la muerte es todo un reto, un tabú moderno que se debe romper para normalizar el contenido afectivo que tiene el fin de la vida para cualquier persona. Es decir, socialmente no estamos preparados para afrontar la muerte y, por este motivo, es un reto difícil y, a su vez, necesario de abordar desde la escolarización obligatoria.

La gran mayoría de pensadores ha reflexionado sobre la finitud de nuestra existencia, la certeza más segura que conoce el ser humano (Esquerda y Agustí, 2010; Mèlich, 2003). En este sentido, de forma implícita, podemos encontrar los antecedentes de la pedagogía de la muerte en los filósofos del siglo iv aC. Pascual (2005) añade que el tema de la educación ocupa un lugar central en el Fedón. Si bien es cierto que no utilizaban el concepto como tal, sí que le buscaban fundamentos y argumentos mediante el diálogo y el debate. El ejemplo más claro lo podemos encontrar en el diálogo que nos proporciona Platón 
en el Fedón, donde Platón se expresa por boca de su maestro, Sócrates, en los momentos previos a la defunción de este último. De hecho, podemos hablar de los inicios más evidentes de la pedagogía de la muerte.

\section{Pedagogía de la muerte en las escuelas}

Las escuelas son espacios vitales de nuestra sociedad. En los centros educativos, suceden, por un lado, acontecimientos que causan goce y felicidad y, por otro lado, hechos que desencadenan la tristeza entre sus miembros (muertes de seres queridos, accidentes, divorcios de los padres, etc.). Estamos convencidos de que no existe una sola escuela o instituto donde no haya una persona en duelo.

En las instituciones docentes, los profesores enseñan multitud de temas transversales, como el reciclaje, la salud, la alimentación, etc. Sin embargo, todavía existe reticencia para tratar en ellas la muerte y la finitud humana (Mèlich, 2003), aunque sean los temas transversales por excelencia. Por el contrario, los maestros que deciden trabajarlos como contenidos transversales del currículum, se encuentran ante un cuestionamiento y una reflexión propia de su estado emocional, de sus creencias, de su experiencia, de sus miedos, de sus incertidumbres, de sus duelos personales, etc. Consecuentemente, el hecho de tratar la finitud humana en el aula requiere un esfuerzo cognitivo y emocional por parte de los profesionales de la educación.

Cuando el centro docente aborda la temática de la muerte, normalmente se está vinculando el contenido de forma explícita con la educación para la salud. Pedrero y Leiva (2011) apuntan que si la salud no solo se entiende como ausencia de enfermedad, sino también como la posesión de un alto grado de calidad de vida (física, mental y social), esta también comporta la educación para la muerte. Los mismos autores denominan este hecho como «tratamiento saludable del tema de la muerte» (Pedrero y Leiva, 2011: 10). De esta forma, seríamos educados para la vida y, así, viviríamos más plenamente, puesto que seríamos conscientes de la finitud humana (Esquerda y Agustí, 2010).

\section{La necesidad de practicar una pedagogía preventiva y normalizadora sobre la finitud humana}

Los niños y las niñas no se están preparando para la vida, pues ya la están viviendo (Kroen, 1996), y para ellos y como parte de la existencia humana, es inevitable encontrarse con la muerte de un ser querido. Además, para velar por el bienestar de las criaturas, es esencial la capacidad de comprender la muerte, atravesar las etapas del duelo y seguir viviendo con eficacia. Es decir, debemos aceptar que la muerte es una realidad de todo ser vivo y, por ello, debemos realizar una pedagogía de la finitud humana. Es por ello que valdría la pena plantearse su integración en el currículo de educación primaria.

En nuestro rol de profesionales de la educación, debemos tener en cuenta que existe una bifurcación didáctica en la enseñanza y en el abordaje pedagógico de la muerte (Herrán y Cortina, 2008): 
- Previa a un acontecimiento trágico. Se desarrolla de forma permanente mediante temas transversales o áreas de conocimiento del currículo.

- Posterior o paliativa. Se lleva a cabo de forma puntual en el momento en que acontece una situación de defunción cercana al niño.

Sin embargo, ambas percepciones tienen continuidad dentro del proceso educativo sobre la muerte. Consideramos firmemente que no es necesario que fallezca un ser querido para que, en consecuencia, se enseñe su contenido y todo aquello que comporta (aspectos sociales, emocionales, biológicos, etc.). En esta línea, Poch y Herrero (2003) añaden que se debe anticipar la muerte y dar respuestas a los niños para que así puedan estar preparados cuando sucedan tales acontecimientos. Tal y como hemos ido comentando, existen otros temas transversales, como, por ejemplo, el reciclaje, la educación vial, la educación para la paz, etc., que se enseñan sin que suceda previamente ningún acontecimiento trágico, como un accidente de tráfico o un conflicto bélico. Desde nuestro punto de vista, creemos que la enseñanza del contenido de la muerte como parte del ciclo vital del ser humano permitirá a los alumnos buscar un sentido a sus existencias de forma paulatina. De este modo, irán apartando las preocupaciones más superfluas para centrarse en aquello que es realmente importante y que tiene como eje el amor.

Cabe añadir que Kübler-Ross (1992, 2013) alegó que se debería preparar a los niños para la muerte antes de experimentarla. Además, tener la oportunidad de anticipar la desaparición de un ser querido es uno de los factores de protección que el entorno puede propiciar para, así, potenciar un duelo positivo en el menor (Díaz Teba, 2004; Fernández Hurtado, 2013). Anticipar el concepto de muerte nos permite dar la oportunidad a los niños de aprender cosas sobre ella sin tener que gestionar, a la vez, todas las emociones que comportan el duelo.

\subsection{Objetivos}

El objetivo general de esta investigación es el de analizar la realidad de la pedagogía de la finitud humana en los centros educativos. Como objetivos específicos, se plantean los siguientes:

a) Conocer cuántos profesionales de la educación, de la psicología, de la pedagogía y/o de la psicopedagogía de los centros seleccionados actuaron frente a una situación infantil o juvenil de muerte y de duelo durante su experiencia profesional.

b) Identificar los motivos por los cuales los profesionales no habían actuado frente a las situaciones antes descritas.

c) Analizar las percepciones y los conocimientos de los profesionales respecto a los materiales, la bibliografía, las herramientas y las estrategias existentes para actuar pedagógicamente en las situaciones de muerte y de duelo en el contexto educativo formal. 


\subsection{Metodología e instrumentalización}

Para realizar el análisis, se utilizó una metodología mixta, con aproximaciones cualitativas y cuantitativas (Bisquerra, 2009) aplicadas al estudio de caso. De este modo, se realizó un estudio de caso (McMillan y Schumacher, 2005), concretamente, de dos centros educativos de la ciudad de Lleida (España): un centro de educación primaria y otro de educación secundaria obligatoria, ambos de titularidad pública. La escuela y el instituto se encuentran situados en el mismo barrio, lo que permitirá también analizar la continuidad de actuaciones en situaciones infantiles y juveniles de muerte y de duelo.

Para la toma de datos, tres expertos diseñaron, realizaron y validaron un cuestionario semiestructurado que estaba diferenciado en cuatro bloques o en cuatro macrocategorías. El primer bloque se centraba en datos generales sobre la pedagogía de la muerte y pretendía captar si el informante se había encontrado con algún caso infantil o juvenil de muerte y de duelo durante su experiencia profesional. Si respondía afirmativamente, la persona debía pasar al segundo bloque del cuestionario; si respondía negativamente, debía ir directamente a la tercera parte del cuestionario. Si el profesional de la educación no había vivenciado ningún caso de muerte y posterior duelo durante su experiencia profesional, el usuario debía pasar directamente a la tercera parte del cuestionario. Es decir, no debía realizar el segundo apartado. Por último, todos los profesionales que contestaron el cuestionario debían responder el último bloque, que hacía referencia a los recursos y a las estrategias bibliográficas. En definitiva, la encuesta era excluyente según las respuestas iniciales del sujeto.

La muestra estaba compuesta por los maestros, los profesores, los pedagogos, los psicopedagogos y/o los psicólogos que estaban en ejercicio en los dos centros educativos, de acuerdo con la descripción que recoge la tabla 1.

Las respuestas de los cuestionarios se complementaron con tres entrevistas semiestructuradas de tipo clínico: dos realizadas a maestras de la escuela (de aquí en adelante Inf_1 i Inf_2) y la restante, a una profesional de la psicología (Inf_3), quien nos facilitará la visión que se debe tener en situaciones de muerte y duelo en el contexto de educación formal.

Tabla 1. Fuentes de información de la investigación realizada

\begin{tabular}{lccc}
\hline \multicolumn{1}{c}{$\begin{array}{c}\text { Fuentes/centros } \\
\text { educativos }\end{array}$} & Maestros y profesores & $\begin{array}{c}\text { Psicopedagogos } \\
\text { y psicólogos }\end{array}$ & Total \\
\hline $\begin{array}{l}\text { Escuela (cuestionario } \\
\text { semiestructurado) }\end{array}$ & 7 & 1 & 8 \\
\hline $\begin{array}{l}\text { Instituto (cuestionario } \\
\text { semiestructurado) }\end{array}$ & 17 & 1 & 18 \\
\hline Total de profesionales & 24 & 2 & 26 \\
\hline
\end{tabular}

Fuente: elaboración propia. 


\subsection{Resultados}

En referencia al cuestionario que se diseñó, analizamos cuatro de las preguntas más significativas que respondieron los maestros, los profesores, los pedagogos, los psicólogos y/o los psicopedagogos de los centros educativos de la muestra. Las contestaciones se complementan con las aportaciones relacionadas de las entrevistas realizadas.

La primera de las preguntas hacía referencia al hecho de si los maestros, los pedagogos, los profesores, los psicopedagogos y/o los psicólogos encuestados se habían encontrado, durante su experiencia profesional, con algún caso infantil o juvenil de muerte y de duelo (gráfico 1).

En la escuela de educación primaria, 5 de los 8 maestros que respondieron el cuestionario se habían encontrado, a lo largo de su experiencia profesional, con algún caso infantil o juvenil de muerte y de duelo, lo que representa el $62,5 \%$ de los profesionales de dicha etapa educativa.

En el caso del instituto analizado, 5 de las 16 personas (un 31,25\%) que respondieron el cuestionario se habían encontrado, a lo largo de su experiencia profesional, con algún caso de duelo juvenil. Asimismo, 4 informantes manifestaron no haberse encontrado con ningún caso sobre la temática que nos ocupa. Cabe señalar que 7 personas del centro de educación secundaria obligatoria manifestaron que no querían responder el cuestionario. Alegaron que no se sentían cómodos con la cuestión que se trataba en él. Este hecho corrobora la tesis de Herrán y Cortina (2006), quienes hicieron patente la incomodidad que sienten muchos docentes cuando tienen que hablar sobre la muer-

Gráfico 1. Total de profesionales que se habían encontrado con algún caso infantil o juvenil de muerte y de duelo durante su experiencia profesional

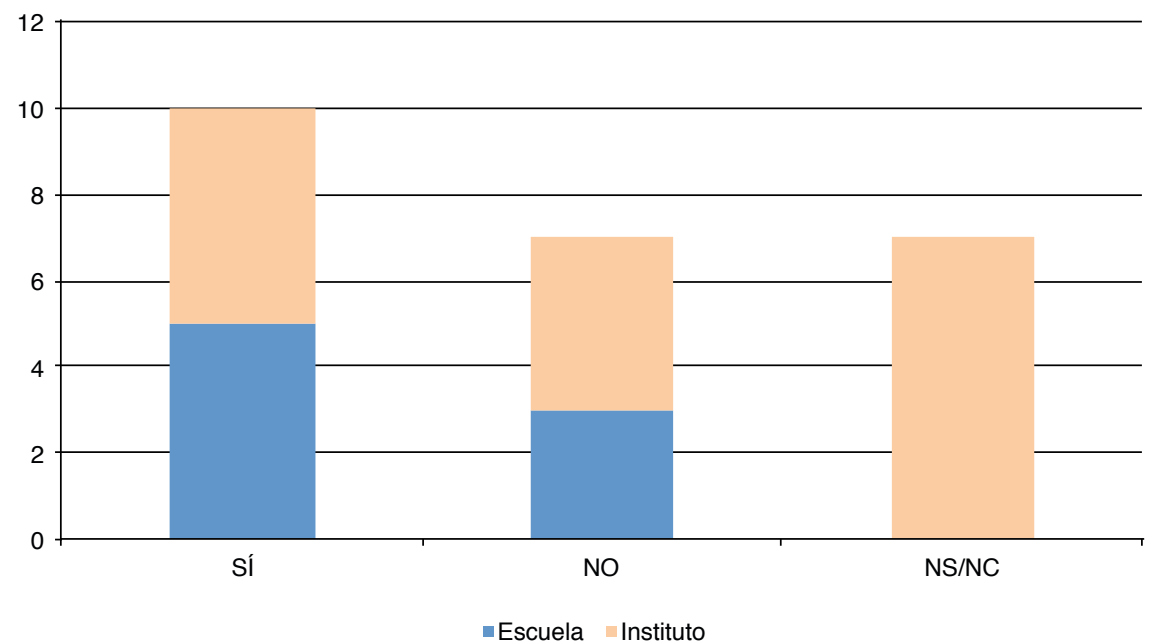

Fuente: elaboración propia. 
te. Las vivencias en relación con la situación descrita se relacionan con las respuestas de la psicóloga entrevistada (Inf_3):

El tabú social que impera sobre la muerte se ha extrapolado a los contextos educativos. Es necesario que los docentes trabajen sus miedos, sus duelos, su concepción sobre la muerte para poder ayudar a los niños a realizar las tareas que requiere un duelo positivo.

Empero, desde la realidad educativa de la escuela, la Inf_2 contrapone la visión de la Inf_3 alegando lo siguiente:

Socialmente, un niño cuando está en la escuela y muere un abuelo, una tía, un hermano, la madre o el padre, se encuentra que esta situación es un hecho íntimo que se vive en casa. Por lo tanto, el cuidado, la comunicación y el sentimiento es mucho más de casa que no de la escuela.

Desde nuestro punto de vista, nos encontramos más en la línea de la Inf_3, ya que la muerte y el duelo son contenidos que es necesario que se trabajen en el ámbito pedagógico, puesto que la escuela es el segundo agente socializador más importante del niño.

La segunda pregunta que debían responder estaba directamente interconectada con la cuestión anterior, al indagar si durante la experiencia profesional de muerte y de duelo infantil y juvenil se actuó pedagógicamente o psicopedagógicamente al respecto (gráfico 2).

Gráfico 2. Total de profesionales que actuaron o que no actuaron pedagógicamente en casos infantiles y juveniles de muerte y de duelo

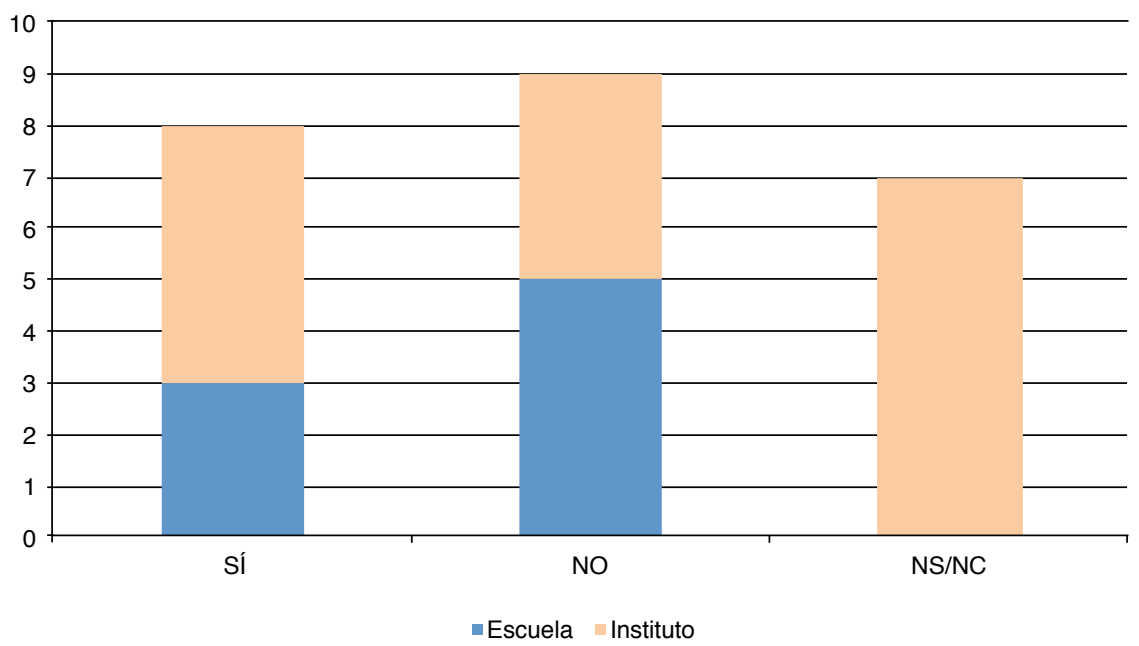

Fuente: elaboración propia. 
Menos de la mitad de los profesionales de la escuela que respondieron el cuestionario (un 37,5\%) actuaron pedagógicamente y/o psicopedagógicamente respecto a las situaciones infantiles y juveniles de muerte y de duelo. En el centro de educación secundaria, actuaron 5 profesionales de los 9 que respondieron el cuestionario. Debemos recordar que, de un total de 16 cuestionarios, solo la mitad de profesionales de la educación secundaria ( 9 sujetos, lo que representa un total de un $56,25 \%$ de la muestra del instituto) quisieron responder. Entre los dos centros de educación formal, 9 profesionales decidieron no actuar en situaciones de muerte y duelo frente a 8 que sí lo hicieron. Los resultados son muy similares si tenemos en cuenta que 7 personas no respondieron.

Uno de los comentarios más significativos de las entrevistas realizadas en referencia a esta pregunta fue el de la Inf_3:

Actualmente nos encontramos situaciones donde las escuelas no explican nada sobre la muerte, aunque los docentes puedan tener en el aula a niños en proceso de duelo.

La tercera pregunta indagaba sobre si los profesionales de la educación, la pedagogía, la psicopedagogía y/o la psicología consideraban que la muerte era una materia para la cual se necesitaban más recursos y estrategias para poder abordarla pedagógicamente y psicopedagógicamente (gráfico 3).

Gráfico 3. Consideraciones sobre la necesidad de destinar recursos pedagógicos para abordar las situaciones infantiles y juveniles de muerte y de duelo

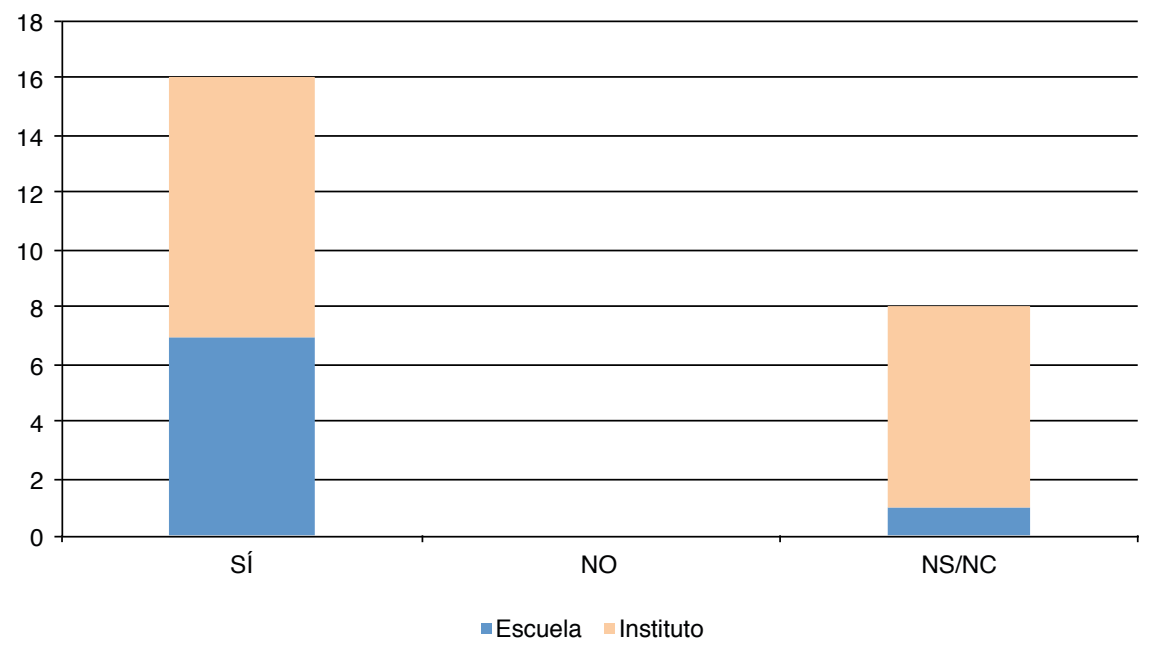

Fuente: elaboración propia. 
Como puede apreciarse en el gráfico 3, 7 de las 8 personas (un 90\%) que respondieron el cuestionario en la escuela consideran que no disponen de suficientes recursos para enseñar o tratar el tema de la muerte en el aula. Por tanto, están de acuerdo en considerar que es necesario disponer de más material pedagógico. Asimismo, un maestro o una maestra no respondió a la pregunta (un 10\%).

También, 9 de los 16 informantes de secundaria manifestaron la necesidad de disponer de recursos y de estrategias para enseñar y tratar la muerte en la etapa adolescente. Este resultado supone un 56,25\% del total de docentes que tuvieron el cuestionario en sus manos, considerando que 7 de las 16 personas no respondieron a esta pregunta (un 43,75\%).

Por lo tanto, nadie explicitó el hecho de la no necesidad de utilizar herramientas y estrategias pedagógicas, pero la no respuesta de la mayoría de los profesionales encuestados da pie a la reflexión. Es decir, podemos pensar que se desconocen los recursos existentes para abordar la temática porque, directamente, no se ha querido actuar al respecto. La causa más clara es el tabú y el rechazo que provoca la muerte entre algunos docentes, sobre todo entre aquellos que no quisieron responder el cuestionario.

Además, uno de los profesores de instituto que sí contestó el cuestionario manifestó lo siguiente al final del documento:

Desde mi punto de vista, creo que, ahora mismo, no estamos preparados para recibir la muerte. Este hecho no es muy comprensible, ya que, por el ritmo natural de la vida, las personas nacemos, crecemos, nos reproducimos o no y morimos. De este modo, debemos reconocer que nos falta formación para este trayecto en el cual finaliza la vida. De todas formas, este tema continúa siendo un tema tabú para la mayoría de personas. Creo que es por la falta de información y formación [...].

Desde las entrevistas, la Inf_1 manifiesta lo siguiente:

En general, los docentes piden la ayuda de una persona que tiene una cierta formación en situaciones como la que aquí nos ocupa. Y, a su vez, es cuando se ve la necesidad de disponer de herramientas pedagógicas que orienten a educadores y a padres.

Es necesario que los recursos pedagógicos para abordar la muerte y el duelo estén disponibles para los centros de educación formal, ya que la finitud de la vida es un hecho inevitable y cotidiano en nuestra existencia.

\subsection{Análisis de resultados}

Desde una perspectiva global, el gráfico 4 recoge la distribución de los informantes por etapa educativa y en relación con su experiencia acerca de la temática. Como podemos observar, 10 profesionales de la educación (de un total de 17 que respondieron) han experimentado en sus clases algún caso infantil o juvenil de muerte y de duelo. Además, cabe señalar que los resultados de esta 
pregunta han sido bastante similares entre los dos centros educativos. Por tanto, vemos que la muerte y el duelo constituyen una realidad con la que los maestros, los profesores, los pedagogos y los psicopedagogos se pueden encontrar en el ámbito formal obligatorio.

Una de las hipótesis que presentamos para poder sostener la igualdad de los resultados obtenidos respecto a las experiencias infantiles y juveniles de muerte y de duelo a lo largo de la vida profesional es el número total de años durante los cuales los profesionales han ejercido activamente. En este sentido, entrarían en juego muchas variables que no analizaremos en este documento, como podrían ser: formación recibida sobre la muerte y el duelo, experiencias personales y profesionales, etc. Asimismo, otra causa sería el tabú que acompaña a la muerte en nuestra sociedad y el choque emocional que comporta dicha temática entre los seres humanos. En este sentido, estudios e investigaciones realizadas por Herrán, González, Navarro, Bravo y Freire (2000) a maestros de educación infantil y psicopedagogos muestran como 2 de cada 3 profesionales entrevistados con más de 5 años de experiencia docente han pasado por situaciones de duelo infantil. Asimismo, otros docentes entrevistados con una gran cantidad de años en ejercicio alegan no haberse enfrentado nunca a una experiencia de muerte ni de duelo infantil. En cambio, detectaron que había algunos que tenían un caso de muerte al año.

En referencia a la pregunta sobre los motivos por los cuales los profesionales de la educación, la psicología, la pedagogía y/o la psicopedagogía no actuaron frente a las situaciones infantiles y juveniles de muerte y de duelo, cabe señalar que había 5 opciones de respuesta incluyendo la categoría «Otros» y

Gráfico 4. Informantes de acuerdo con la experiencia en casos infantiles y juveniles de muerte y de duelo

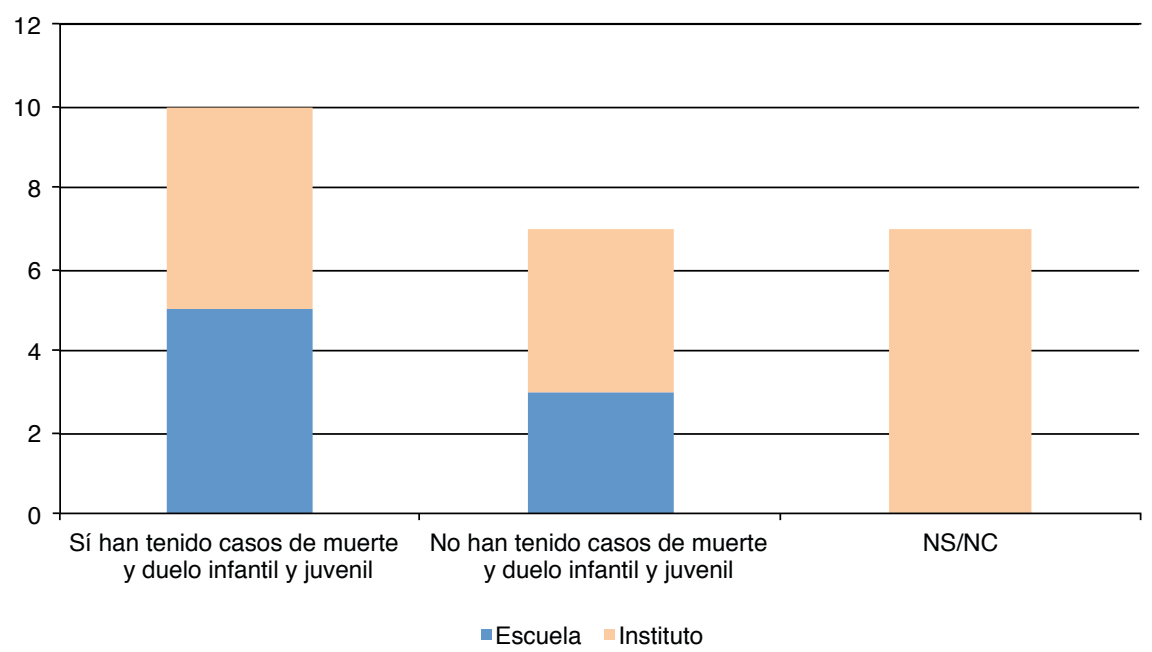

Fuente: elaboración propia. 
que no eran excluyentes entre sí. Las opciones que se propusieron fueron: «Desconocimiento del tema», "Desconocimiento de estrategias de actuación» (Selfa, Fraga y Berengué, 2014), «No quise hacer nada» $\mathrm{y}$ «El equipo directivo no quiso actuar al respecto». En el centro de educación primaria, 3 personas respondieron que fue por desconocimiento de estrategias de actuación y 2 no supieron o no quisieron contestar. En secundaria, 2 profesionales marcaron la opción «Desconocimiento de estrategias de actuación»; 3, la opción «No quise hacer nada», $y$, finalmente, 4 alegaron "El equipo directivo no quiso actuar al respecto». La interpretación de estos resultados se incluye en la pregunta siguiente y se relaciona con ella. Por lo que se refiere a la existencia de recursos pedagógicos sobre la temática, 15 personas del total de 24 que respondieron esta pregunta consideran que no existen suficientes recursos bibliográficos. El profesional que no contestó esta pregunta escribió, en el mismo papel del cuestionario, que desconocía el tema (gráfica 5). Cabe añadir que 10 personas del total de 18 (un 55,55\%) del centro de educación secundaria obligatoria no la respondieron. En ese sentido, apelamos a la hipótesis que hemos mencionado antes, aunque apostamos firmemente por el miedo y el rechazo que provoca la muerte en la sociedad en general.

La existencia de recursos bibliográficos para trabajar dicha temática en el aula es reconocida como escasa. Esta consideración se puede relacionar con la anterior referida a los motivos por los cuales no se actuó en situaciones infantiles y juveniles de muerte y de duelo. Consideramos que todavía no existen muchos recursos pedagógicos ni formación suficiente del profesorado sobre esta cuestión. Este hecho se puede contrastar fácilmente con la gran cantidad

Gráfico 5. Informantes de acuerdo con la necesidad de disponer de recursos

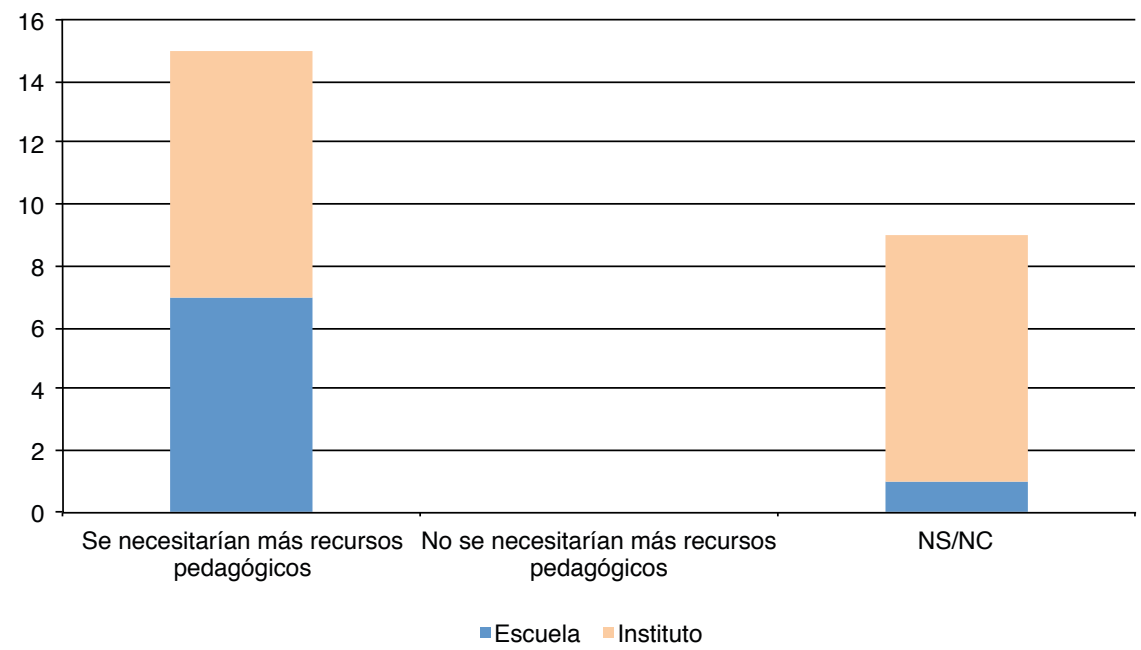

Fuente: elaboración propia. 
y calidad de materiales que abordan otras áreas del conocimiento del currículo, ya sea de educación primaria o de educación secundaria; por ejemplo: los recursos y las herramientas existentes para practicar las operaciones con figuras geométricas.

Como consecuencia de todo lo que hemos ido comentando, apostamos por la necesidad de diseñar un programa pedagógico preventivo y normalizador sobre la muerte para abordar este acontecimiento antes de que suceda. De este modo, los niños tendrán más herramientas para afrontar el conjunto de emociones que se desarrollan a partir de una pérdida afectiva. Este hecho lo manifiesta la Inf_2:

Yo creo que sería muy importante diseñar un programa pedagógico preventivo sobre la muerte.

\section{Conclusiones}

\subsection{Respecto a los objetivos de la investigación}

La pedagogía de la muerte es un tema complejo que exige abordar educativamente la finitud humana desde la escuela. Dicha temática como fuente de sabiduría sobre la vida debe tratarse siempre y cuando se aborde desde el respeto, la conciencia y la tolerancia. Se trata de romper con la clásica mentalidad negativa sobre la muerte, que la concibe como un sufrimiento y un fin sin sentido. Si se abordara el tema pedagógicamente y se tomara conciencia progresiva de la finitud humana, se podrían obtener resultados terapéuticos. Asimismo, se debe considerar que, a largo plazo, un duelo mal resuelto puede comportar problemas como depresión, estrés, ansiedad, etc.

El estudio realizado confirma la necesidad de hablar de la finitud humana en la educación formal. Tal y como hemos comentado, los profesionales de la educación, de la psicología, de la pedagogía y/o de la psicopedagogía que han respondido el cuestionario y las entrevistas realizadas corroboran y confirman la necesidad señalada, aunque, de acuerdo con Ramos Álvarez (2014), como profesionales de la docencia, no quieran o no se atrevan a intervenir en casos infantiles y juveniles de muerte y de duelo. Estamos convencidos de que el motivo clave por el cual los educadores no quieren actuar frente a dichas situaciones es la concepción general que se tiene de la muerte. Es decir, si, como seres humanos, queremos eludir la finitud de nuestra existencia, es porque omitimos y escapamos de toda situación y emoción que produzca angustia, miedo o sufrimiento, lo que refleja el claro tabú donde se sitúa la muerte en nuestra sociedad occidental.

Las respuestas de los profesionales educativos, de la psicología, de la pedagogía y/o de la psicopedagogía varían en función de si ejercen profesionalmente en la escuela o en el centro de educación secundaria. La mayoría considera que no existen suficientes recursos bibliográficos que traten sobre la finitud de la vida y sobre el duelo infantil y juvenil, si bien están surgiendo iniciativas de expertos en la muerte y el duelo en España, quienes proponen estrategias, actividades y 
herramientas para afrontar dichas situaciones desde el respeto, la alteridad y la comprensión. Creemos que la pedagogía de la finitud y la muerte - la una implica a la otra- es una necesidad formativa de la carrera de Magisterio.

\subsection{Respecto a las debilidades metodológicas del estudio}

Este estudio de caso no pretende ser ninguna generalización. Los datos manejados son escasos, pero han permitido adquirir una primera percepción de la concepción que tienen los profesionales de la educación sobre la muerte. Por este motivo, posteriormente expondremos propuestas de futuro.

\subsection{Respecto a las necesidades formativas de los maestros, de los profesores, de los psicólogos, de los pedagogos y de los psicopedagogos}

La revisión de la literatura y el análisis de las aportaciones de los profesionales nos permiten proponer algunas actividades para su formación y, si cabe, también la de las familias, así como una serie de actividades para tratar la muerte y el duelo en las aulas desde una perspectiva general (adaptado de Rodríguez Herrero, Herrán y Cortina, 2012: 181):

- Coloquios, conferencias, mesas redondas y/o paneles. En estas actividades, se puede plantear la temática de la muerte como eje transversal del debate $y$, a partir de aquí, hablar y debatir sobre los diferentes enfoques existentes. De esta forma y contrastando con las diferentes ideas, opiniones y creencias, se puede tomar conciencia de la importancia de la pedagogía de la muerte.

- Entrevistas públicas colectivas con expertos. Desde la visión de un profesional experto en las temáticas de la muerte y el duelo entre la población escolar infantil y juvenil, también se puede tomar conciencia de la necesidad que tienen los centros educativos de tratar dichas situaciones. Además, el experto puede aportar experiencias y estrategias para realizar actividades anticipantes y/o paliativas.

- Desde una visión anticipante, se puede invitar a los adolescentes de los centros de educación secundaria a intervenir en estos debates y coloquios, aunque no tienen por qué participar activamente en ellos si no quieren o no se sienten cómodos. Debemos recordar que los adolescentes tienen el pensamiento cognitivo formal y, por ello, disponen de la capacidad de razonar y reflexionar sobre conocimientos abstractos. Por este motivo creemos que, desde la libertad de actuación, se debe dejar asistir a los alumnos a este tipo de actividades.

- Actividades anticipantes del miedo. Esta tipología de tareas se puede empezar a trabajar desde el primer ciclo de educación primaria e ir ampliando sus contenidos de forma progresiva. En este sentido, el miedo es uno de los subconceptos que implican la concepción de la muerte.

- Rincones de actividad. Esta tipología de trabajo se practica habitualmente a lo largo de la educación primaria. En dicho sentido, proponemos realizar 
actividades relacionadas con fotografías antiguas de los familiares para relacionar los conceptos de vivo y de muerto o también analizar seres vivos de la naturaleza, como, por ejemplo, hojas de árboles diferentes (perennes y caducas). En educación secundaria, podemos proponer que los alumnos analicen, por grupos de trabajo, diferentes medios de comunicación para estudiar la difusión y el trato que recibe la muerte en ellos.

- Áreas curriculares y unidades didácticas. Se puede incorporar el contenido de la muerte y la finitud humana en las sesiones de tutoría, pero ello supondría un tratamiento sesgado y dicotomizado del resto de las áreas curriculares. Desde nuestro parecer y de acuerdo con Rodríguez Herrero, Herrán y Cortina (2012), la pedagogía de la muerte es y debe ser un eje transversal y radical en la educación, por lo que es importante apostar por una visión transdisciplinaria y transversal del currículo.

- Proyectos pedagógicos y pequeñas investigaciones temáticas. Esta tipología de proyectos permite interconectar diversas áreas curriculares en la enseñanza de la muerte como contenido normalizado de los seres humanos. Podemos incorporar y vincular tradiciones de nuestra sociedad como el entierro de la sardina, el Día de los Santos Inocentes, el Día de Todos los Santos, etc. Además, podemos ampliar nuestra visión como ciudadanos de un mundo global y apostar por el desarrollo integral del alumnado mediante la curiosidad de los niños y de los adolescentes respecto a otras culturas, trabajando pedagógicamente, por ejemplo, el Día de los Muertos, que se celebra en Méjico.

- Metáforas, analogías y cuentos. Podemos utilizar estos recursos pedagógicos para introducir el concepto de muerte de forma progresiva en el aula, teniendo en cuenta el desarrollo cognitivo de los alumnos. Existe, por ejemplo, una amplia variedad de cuentos que permiten trabajar la pedagogía de la muerte desde que los niños son conscientes de que esta existe, aunque no conozcan sus consecuencias plenamente. Colomo $(2015,2016)$ añade que dichas narraciones sirven para reflexionar sobre las experiencias de cada uno, para reconstruir el mundo interno de las personas y, a su vez, aumentar la confianza mediante la identificación con los personajes que aparecen en ellos. Además, el mismo autor afirma que el lugar ideal para tratar los cuentos es el aula escolar, ya que se trata del sitio de «ensayo para la vida». Cabe añadir que tenemos constancia de una experiencia muy positiva en educación infantil con cuentos (Selfa, Fraga y Berengué, 2014), donde se intervino a partir de un plan de diálogo y se trabajó la comprensión literal del texto y la muerte a partir de una narración sobre esta. Mediante la biblioterapia, se puede hacer frente a acontecimientos muy significativos de forma satisfactoria, como, en este caso, el final de la vida humana.

- Acompañamiento empático. Para poder asegurar un proceso de duelo positivo y terapéutico, Bowlby (1983) introdujo las primeras nociones para lograrlo. Abogó por la importancia de poder ser acompañado en todo momento por alguien cercano que pueda comprender el duelo y que ponga 
palabras al dolor que se siente. Además, debemos escuchar al otro desde el silencio y la comprensión, sin juzgar ni dar nada por supuesto.

\section{Propuestas de futuro}

Las propuestas que se realizan parten del posicionamiento en contra del «taponamiento" que sufre la muerte en nuestra sociedad occidental. Además, este hecho es más pronunciado cuando se trata de niños, aunque, a nuestro parecer, muerte e infancia no son palabras antagónicas. A su vez, creemos firmemente que, mediante la innovación pedagógica en la enseñanza de la finitud humana, podemos progresar como sociedad, sobre todo si actuamos desde la escuela. Debemos recordar que los centros educativos son los agentes socializadores más tempranos e importantes para los niños y adolescentes.

Además, se pueden plantear algunas cuestiones en relación con la temática tratada. En primer lugar, cabe preguntarse por qué una cantidad considerable de profesionales de la educación, la psicología, la pedagogía y/o la psicopedagogía del centro de educación secundaria no responde cuando se le pide si, en él, se actuó pedagógicamente en situaciones infantiles y juveniles de muerte y de duelo. Asimismo, debemos de continuar trabajando para obtener datos que permitan clarificar el motivo de la baja participación en el cuestionario por parte de los docentes, los pedagogos y el psicopedagogo y/o el psicólogo del instituto, a fin de profundizar sobre las concepciones que tienen los profesionales de la educación sobre la muerte, tanto la ajena como la propia.

Por otro lado, se necesitaría reflexionar más sobre los motivos por los cuales los profesionales que actuaron respecto a las situaciones infantiles y juveniles de muerte y de duelo afirman que no buscaron, según confiesan ellos mismos, recursos ni estrategias bibliográficas de forma suficiente.

La investigación realizada se puede llevar a cabo en contextos más amplios. Además, las estrategias y las herramientas de análisis pueden ser más extensas y exhaustivas con una muestra de población mayor. Es decir, no es necesario limitarse a realizar estudios de casos puntuales.

Finalmente, apostamos por profundizar en la naturaleza, en el sentido y en el significado de una pedagogía preventiva y normalizadora sobre la finitud humana, considerando que esta alternativa constituye la clave para la educación plena de los niños y los adolescentes mediante la normalización de la muerte (Cantero, 2013; Gabarró, 2016). Esta opción no es incompatible con la pedagogía paliativa, sino que más bien la completa si tratamos la muerte con normalidad, desde los centros educativos, como acontecimiento inevitable de la vida, y cambiamos poco a poco las concepciones negativas que se suelen tener sobre el acabamiento de nuestra existencia. Una vida experienciada plenamente, con absoluta conciencia de nuestra finitud, puede suponer una experiencia rica, tanto desde el punto de vista pedagógico como emocional. 


\section{Referencias bibliográficas}

ArIÉs, P. (1975). Historia de la muerte en occidente: Desde la edad media hasta nuestros dias. Madrid: Acantilado.

Arnaiz, V. (2003). ¿Podemos hablar de la muerte en la escuela y en el instituto? Aula de Innovación Educativa, 122, 36.

Bisquerra, R. (2009). Metodología de investigación educativa. Madrid: La Muralla.

Bowlby, J. (1983). El apego y la pérdida III: La pérdida. Barcelona: Paidós.

Cantero, M.F. (2013). La educación para la muerte: Un reto formativo para la sociedad actual. Psicogente, 16(30), 424-438.

Carmelo, A. y Comas, K. (2014). ¿Existe la muerte?: Ciencia, vida y trascendencia. Barcelona: Plataforma.

Colomo, E. (2015). Diversidad cultural y educación. Learning \& Pedagogics, 1, 12-19.

- (2016). Pedagogía de la muerte y proceso de duelo: Cuentos como recurso didáctico. Revista Iberoamericana sobre Calidad, Eficacia y Cambio en Educación, 14(2), 63-77.

Colomo, E. y Oña, J.M. de (2014). Pedagogía de la muerte: Las canciones como recurso didáctico. REICE. Revista Iberoamericana sobre Calidad, Eficacia y Cambio en Educación, 12(3), 109-121.

Cuadrado, L. (productor) y Manzano, E. (dir.) (2016). Amb filosofia: La mort [vídeo]. Barcelona: Televisió de Catalunya (TV3).

DíAz TebA, I. (2004). I ara, on és?: Com ajudar els nens $i$ els adolescents a entendre la mort. Barcelona: Oxigen Viena.

Esquerda, M. y Agustí, A.M. (2010). El nen i la mort: Acompanyar els infants $i$ adolescents en la pèrdua d'una persona estimada. Lleida: Pagès Editors.

Fernández Hurtado, I. (2013). El Joan ha mort: Conte i guía per acompañar els nens $i$ els adolescents en el dol $i$ la comprensió de la mort. Lleida: Pagès Editors.

Gabarró, D. (2016). Explicar la muerte a niños/as y adolescentes 5/6. Recuperado de $<$ http://campus.danielgabarro.com/cursos-para-ninos-y-ninas/explicar-la-muertea-ninosas-y-jovenes/explicar-la-muerte-a-ninosas-y-jovenes-6>.

Herrán, A. y Cortina, M. (2006). La muerte y su didáctica: Manual para Educación Infantil, Primaria y Secundaria. Madrid: Humanitas.

- (2008). La Práctica del «Acompañamiento Educativo» desde la Tutoría en Situaciones de Duelo. Tendencias Pedagógicas, 13, 157-173.

Herrán, A.; González, I.; Navarro, M.J.; Bravo, S. y Freire, M.V. (2000). ¿Todos los caracoles se mueren siempre?: Cómo tratar la muerte en educación infantil. Madrid: Ediciones de la Torre.

Kroen, W. (1996). Cómo ayudar a los niños a afrontar la pérdida de un ser querido. Barcelona: Paidós.

KüBleR-Ross, E. (1992). Todo final es un luminoso principio. Barcelona: Sirpus.

- (2013). La mort: una aurora. Barcelona: Luciérnaga.

McMillan, J.H. y Schumacher, S. (2005). Investigación educativa: Una introducción conceptual. Madrid: Pearson Educación.

Mèlich, J.M. (2003). Por una pedagogía de la finitud. Aula de Innovación Educativa, $122,39-40$.

Olid BÁEz, I. (2013). ¿Por qué una tesis sobre la muerte?: Entrevista con Txabi Arnal Gil. Bellaterra Journal of Teaching \& Learning Language \& Literature, 6(1), 72-80.

Otero, I. y Soares, F. (2012). Pedagogía de la muerte en el nivel de enseñanza primaria. Revista Iberoamericana de Educación, 60(3), 1-8. 
Pascual, F. (2005). Esperanza y escatología: Diálogo entre la fe y la razón. Spe Salvi: Veritas, 3(18), 47-61.

Pedrero, E. y Leiva, J.J. (2011). La muerte: tema radical y perenne en la educación: Hacia una (R)evolución educativa. XII Congreso Internacional de Teoría de la Educación, $1-13$.

Plató (1999). Fedó. Barcelona: Edicions 62.

Poch, C. y Herrero, O. (2003). La muerte y el duelo en el contexto educativo: Reflexiones, testimonios y actividades. Barcelona: Paidós.

Ramos Álvarez, R. (2014). Las estrellas fugaces no conceden deseos: Programa de prevención, evaluación e intervención por duelo en el contexto escolar. Madrid: TEA Ediciones.

Rodríguez Herrero, P.; Herrán, A. de la y Cortina, M. (2012). Antecedentes de Pedagogía de la Muerte en España. Enseñanza \& Teaching, 30(2), 175-195.

- (2015a). Pedagogía de la muerte mediante aprendizaje de servicio. Educación XX1, 18(1), 189-212.

- (2015b). Educar y vivir teniendo en cuenta la muerte: Reflexiones y propuestas. Madrid: Pirámide.

Selfa, M.; Fraga, F. y Berengué, I. (2014). Leer sobre la muerte en la Biblioteca de Aula: Una experiencia práctica de lectura en un aula de educación infantil. Investigaciones sobre Lectura, 3, 83-95.

SerRa Llanas, X. (2014). I jo, també em moriré?: Com es pot ajudar els infants $i$ els joves a conviure amb la pèrdua i la mort de qui estimem. Barcelona: Columna. 